\title{
Erratum: Infantile amnesia reflects a developmental critical period for hippocampal learning
}

Alessio Travaglia, Reto Bisaz, Eric S Sweet, Robert D Blitzer \& Cristina M Alberini

Nat. Neurosci. 19, 1225-1233 (2016); published online 18 July 2016; corrected after print 29 August 2016

In the version of this article initially published, $y$-axis labels in Figures 3a,b, $4 c, 5 \mathrm{~d}$ and $6 \mathrm{c}$ report the "GluN2B/GluN2A ratio"; this should be

"GluN2A/GluN2B ratio." The error has been corrected in the HTML and PDF versions of the article.

\section{Corrigendum: Opportunities and challenges in modeling human brain disorders in transgenic primates}

Charles Jennings, Rogier Landman, Yang Zhou, Jitendra Sharma, Julia Hyman, J Anthony Movshon, Zilong Qiu, Angela Roberts, Anna Wang Roe, Xiaoqin Wang, Huihui Zhou, Liping Wang, Feng Zhang, Robert Desimone \& Guoping Feng Nat. Neurosci. 19, 1123-1130 (2016); published online 26 August 2016; corrected after print 29 August 2016

In the version of this article initially published online, the first author's name appears as "Charles Jennings" without middle initial; it has been changed to "Charles G Jennings". Another author's name appears in the author list as "Angela Roberts," also without middle initial; it has been changed to "Angela C Roberts." The error has been corrected in the PDF and HTML versions of this article.

\section{Corrigendum: A viral strategy for targeting and manipulating interneurons across vertebrate species}

Jordane Dimidschstein, Qian Chen, Robin Tremblay, Stephanie L Rogers, Giuseppe-Antonio Saldi, Lihua Guo, Qing Xu, Runpeng Liu, Congyi Lu, Jianhua Chu, Michael C Avery, Mohammad S Rashid, Myungin Baek, Amanda L Jacob, Gordon B Smith, Daniel E Wilson, Georg Kosche, Illya Kruglikov, Tomasz Rusielewicz, Vibhakar C Kotak, Todd M Mowery, Stewart A Anderson, Edward M Callaway, Jeremy S Dasen, David Fitzpatrick, Valentina Fossati, Michael A Long, Scott Noggle, John H Reynolds, Dan H Sanes, Bernardo Rudy, Guoping Feng \& Gord Fishell

Nat. Neurosci. 19, 1743-1749 (2016); published online 31 October 2016; corrected after print 29 November 2016

In the version of this article initially published, authors Joshua S. Grimley, Anne-Rachel Krostag and Ajamete Kaykas were missing. These authors have been inserted into the author list after Jianhua Chu; they are at the Allen Institute for Brain Science, Seattle, Washington, USA, and performed experiments related to hESCs. The error has been corrected in the HTML and PDF versions of the article.

\section{Addendum: A viral strategy for targeting and manipulating interneurons across vertebrate species}

Jordane Dimidschstein, Qian Chen, Robin Tremblay, Stephanie L Rogers, Giuseppe-Antonio Saldi, Lihua Guo, Qing Xu, Runpeng Liu, Congyi Lu, Jianhua Chu, Michael C Avery, Mohammad S Rashid, Myungin Baek, Amanda L Jacob, Gordon B Smith, Daniel E Wilson, Georg Kosche, Illya Kruglikov, Tomasz Rusielewicz, Vibhakar C Kotak, Todd M Mowery, Stewart A Anderson, Edward M Callaway, Jeremy S Dasen, David Fitzpatrick, Valentina Fossati, Michael A Long, Scott Noggle, John H Reynolds, Dan H Sanes, Bernardo Rudy, Guoping Feng \& Gord Fishell

Nat. Neurosci. 19, 1743-1749 (2016); published online 31 October 2016; corrected after print 29 November 2016; addendum published after print 29 May 2017

The authors wish to acknowledge a potentially relevant work that they were made aware of after publication: Lee, A.T., Vogt, D., Rubenstein, J.L. \& Sohal, V.S. A class of GABAergic neurons in the prefrontal cortex sends long-range projections to the nucleus accumbens and elicits acute avoidance behavior. J. Neurosci. 34, 11519-11525, http://dx.doi.org/10.1523/JNEUROSCI.1157-14.2014 (2014). This work describes an approach for using Dlx1/2 enhancers to attempt to achieve selective expression in cortical GABAergic interneurons. Although thorough validation was not performed, the results are consistent with the possibility that other regulatory elements may be generalizable as an effective way of targeting specific cell types. 